\title{
Young's modulus of phyllosilicate nanoscrolls measured by the AFM and by the in-situ TEM indentation
}

\author{
M. M. Khalisov ${ }^{1,2}$, V. A. Lebedev ${ }^{3}$, A. S. Poluboyarinov ${ }^{4}$, A. V. Garshev ${ }^{4}$, \\ E. K. Khrapova ${ }^{1}$, A. A. Krasilin ${ }^{1}$, A. V. Ankudinov ${ }^{1}$ \\ ${ }^{1}$ Ioffe Institute Politekhnicheskaya, 26, Saint-Petersburg 194021, Russian Federation \\ ${ }^{2}$ Pavlov Institute of Physiology, Russian Academy of Sciences \\ Makarova emb., 6, Saint-Petersburg, 199034, Russian Federation \\ ${ }^{3}$ University of Limerick Limerick, V94 T9PX, Ireland \\ ${ }^{4}$ Lomonosov Moscow State University GSP-1, Leninskie Gory, Moscow, 119991, Russian Federation \\ Alexander.ankudinov@mail.ioffe.ru
}

PACS 07.79.Lh, 87.64.Ee

DOI 10.17586/2220-8054-2021-12-1-118-127

\begin{abstract}
$\mathrm{Ni}_{3} \mathrm{Si}_{2} \mathrm{O}_{5}(\mathrm{OH})_{4}$ phyllosilicate nanoscrolls were investigated by two techniques: the bending-based test method of AFM and the indentation method with visual control in STEM. In the first case, the average measured Young's modulus, about 200 GPa, turned out to be significantly higher than in the second one, $40 \mathrm{GPa}$. The reasons for this discrepancy are analyzed.
\end{abstract}

Keywords: AFM, in-situ TEM, nanomechanics, indentation, Young's modulus.

Received: 10 December 2020

Revised: 1 February 2021

\section{Introduction}

Phyllosilicates with chrysotile, pecoraite, halloysite structure, as well as some others [1] roll up into long nanotubes and nanoscrolls with outer and inner diameters being in the range of $20-200 \mathrm{~nm}$ and $4-30 \mathrm{~nm}$, respectively. The driving forces of the phyllosilicate layers scrolling are the surface structure differences and the crystal lattice mismatches between the metal-oxygen (octahedral) and silicon-oxygen (tetrahedral) sheets [2]. A similar mechanism underlies the method of 3D micro- and nanostructures production [3], in which a strained semiconductor film is separated from the substrate using a sacrificial layer, and then scrolled to compensate the strains. Scrolled structures may also be formed from a thin, unstressed film to compensate the surface energy difference of the top and bottom sides of the film, [4]. Owing to their composition, structure and morphology, phyllosilicate nanoscrolls are considered as promising adsorbents and capsules [5-7], catalysts [8-12] and reinforcing components of composite materials [13-16]. In the view of their latter application, it seems interesting and important to determine the mechanical properties of the individual phyllosilicate nanoscrolls.

The morphology, composition, structure, and mechanical properties of individual nanoobjects can be studied using the methods of scanning and transmission electron microscopy (SEM, TEM), as well as atomic force microscopy (AFM) [17-19]. The bending-based test method of AFM [20-22] provides an opportunity to measure the Young's modulus of tubes, rods, and scrolls. For such a quasi-one-dimensional object, a nanobridge, bending stiffness is determined, and the result is analyzed using the theory of elasticity $[23,24]$. The nanobridges are formed, for example, over the pores of the track membrane after a droplet of colloid of one-dimensional nanoobjects has dried [25], or by using other sample preparation methods [26]. For nanoobjects located on a solid substrate, the Young's modulus can also be measured using the novel indentation technique with in-situ detection of the actual object's deformation by TEM or SEM [27].

In this study, aimed at determining the Young's modulus of synthetic nanoscrolls $\mathrm{Ni}_{3} \mathrm{Si}_{2} \mathrm{O}_{5}(\mathrm{OH})_{4}$ with a pecoraite structure, the capabilities of the AFM and the in-situ TEM indentation techniques are compared.

\section{Sample preparation and investigation methods}

The $\mathrm{Ni}_{3} \mathrm{Si}_{2} \mathrm{O}_{5}(\mathrm{OH})_{4}$ phyllosilicate nanoscrolls were synthesized by hydrothermal treatment at $350^{\circ} \mathrm{C}$ of the product of the reverse precipitation reaction from $\mathrm{NiCl}_{2}$ and $\mathrm{Na}_{2} \mathrm{SiO}_{3}$ solutions, the procedure details are given in [28].

TEM data were collected at $200 \mathrm{kV}$ in HAADF-STEM mode using Libra 200 microscope (Zeiss, Germany). To minimize undesired charging effects, beam monochromatization was employed. For the in-situ TEM indentation, Hysitron PI-95 picoindenter (Bruker, USA) was implemented. This setup permits to control tip displacements and the 
force applied with a high precision using the embedded actuator. Moreover, series of (S)TEM images acquired during the measurements allows one to observe the entire indentation process.

Sample preparation was performed as follows: a droplet of a suspension of particles in isopropyl alcohol was applied to silicon substrates with a trapezoidal microprotrusion with a top base width of $1 \mu \mathrm{m}$ (Flat-top silicon wedges; Bruker, USA) and then dried in air. The substrates were attached with conductive paste to a copper prism which than mounted into the Hysitron PI-95. The force applied by the indenter was pre-calibrated on a microcantilever with a known stiffness, $45 \mathrm{~N} / \mathrm{m}$. Loading and unloading forces as functions of sample deformation were corrected for drift in accordance with TEM data. This made it possible to determine the position of the picoindenter tip with an accuracy sufficient to investigate samples $20-50 \mathrm{~nm}$ thick.

The indentation data processing was carried using the Hertz contact theory. The selected section of the corrected force curve was approximated by the dependence of this theory for the paraboloid-cylinder contact:

$$
F=\frac{4}{3} E^{*} \sqrt{\frac{R^{*}}{\gamma}} \delta^{\frac{3}{2}} .
$$

The following designations are used. Effective Young's modulus $E^{*}=\left(\left(1-\nu_{P}^{2}\right) / E_{T}+\left(1-\nu_{S}^{2}\right) / E_{S}\right)^{-1}$; reduced radius $R^{*}=R_{T} R_{S} /\left(R_{T}+2 R_{S}\right)$ and the ellipticity parameter $\varepsilon=\left(R_{S}+R_{T}\right) / R_{S}$ characterize the bodies in contact; $\gamma \approx 2(1+0.4 \ln \varepsilon)^{3} \varepsilon^{-0.27} /(3 \varepsilon+1.8)$; load force $F, \delta$ is the total deformation of the nanoscroll and the diamond indenter (we neglected the latter); $R_{S}$ and $R_{T} \approx 100 \mathrm{~nm}$ are the radii of cylindrical nanoscroll, sample (S), and parabolic indenter (T); $E_{S}$ and $E_{T}=1140 \mathrm{GPa}$ are the sample and indenter Young's moduli; Poisson's ratios $\nu_{S}=\nu_{T} \approx 0.3$. Eq. (1) were obtained for an axially symmetric indenter and when the length of the cylindrical sample is significantly greater than its radius. The latter makes it possible to neglect the sample-substrate contact's deformation, see Appendix.

Several samples for TEM experiments were studied by AFM on an NTEGRA Aura setup with Nova Px control program and HybriD Mode of the operation (NT-MDT SI, Russia).

To prepare the samples with nanobridges for AFM testing, a suspension of $\mathrm{Ni}_{3} \mathrm{Si}_{2} \mathrm{O}_{5}(\mathrm{OH})_{4}$ nanoscrolls in isopropanol with a concentration of about $0.3 \mathrm{~g} / \mathrm{l}$ was used. A droplet of the suspension was applied and dried on a TGZ3 silicon calibration grating (NT-MDT SI) with a period of $3 \mu \mathrm{m}$ and a rectangular groove depth of $558 \mathrm{~nm}$. The advanced version of the bending-based test method of AFM was used with special algorithm to establish the nanobridge span length and to identify the boundary conditions of the nanobridge fixation [29]. If one end of the nanobridge rested on a protrusion and the other was in a depression, we used the results of [30] to correct the contribution to the deformation signal from an AFM probe sliding over an inclined object. The nanobridges, formed from nanoscrolls deposited on the gratings, were preliminarily studied in a Quanta 200 scanning electron microscope (FEI, USA). Subsequently, SEM data were used to facilitate the detection of the nanobridges in a BioScope Catalyst atomic force microscope (Bruker, Germany) integrated into a Z16 APO optical microscope (Leica, Germany).

The bending-based tests were conducted in the PeakForce QNM AFM mode, simultaneously registering the signals of the sample relief height, the deformation $D$, and the peak force error $F_{E}$. The signals were determined by an automatic force curve analysis algorithm in the scanning program. To visualize and analyze the AFM data, the NanoScope Analysis 1.80 (Bruker) program and free software Gwyddion version 2.55 were used [31].

In the signal $D$, the instrumental contributions were corrected. Automatically, the deformation is determined by $85 \%$ of the contact part of the force curve and does not correspond to the setpoint force $F_{S P}$, but to the actual force, which is the sum of $F_{S P}$ and $F_{E}$. To eliminate the contribution from the AFM probe sliding on inclined sample areas into the signal $D$, we used a simplified version of the filter [30], which is reduced to multiplying the measured signal value by the square of the cosine of the angle $\theta$ between the vertical direction and the surface normal at the point of measurements. Such a correction does not affect the bending profile of horizontally located nanobridges, since it is equal to unity on them. The corrected deformation was calculated, thus, by the expression:

$$
D_{C}=\frac{D F_{S P} \cos ^{2} \theta}{0.85 F_{S P}+0.85 F_{E}} .
$$

Two nanobridge bending profiles were extracted from the AFM image of $D_{C}$ values: (1) with the nanobridge span length (the distance between the attachment points at the boundaries of the calibration grating groove) $l_{T}$, determined from the AFM topography data; (2) with a span length $l_{S}$, the start and end points of which were set at the locations where the $D_{C}$ signal began to exceed the horizontal baseline of zero signal.

Then the profiles (1) and (2) were normalized vertically, $\zeta=D_{C} / D_{C}^{M A X}$, and horizontally, $\chi=x / l_{i}(x$ is the coordinate value along the profile, $i=T, S$ ), and approximated by the expression:

$$
\zeta_{n}(\chi)=4^{n}\left(\chi-\chi^{2}\right)^{n}, \quad \chi \in[0,1]
$$


The fitting parameter $n$ describes the model of deformation and fixation of the nanobridge at the edges of the depression. For $n=1$, the object under study exhibits the properties of a stretched string, for $n=2-$ a supported beam, for $n=3-$ a clamped beam [29].

From the two profiles with $2 \leq n \leq 3$, the one with a minimum residual with the fit, Eq. (3), was selected, i.e. more consistent with the theory [29], which was then approximated by the expression:

$$
\zeta^{\lambda}(\chi)=4^{3}\left(\chi-\chi^{2}\right)^{3} \frac{(2+\lambda)}{(1+2 \lambda)(2+3 \lambda)}+4^{2}\left(\chi-\chi^{2}\right)^{2} \frac{6 \lambda(\lambda+1)}{(2 \lambda+1)(3 \lambda+2)} .
$$

Young's modulus, $E$, was calculated using the $\lambda$ value, which characterizes the nanobridge fixing conditions and is the result of fitting [29]:

$$
E=\Phi(\lambda) E_{C B} ; \quad E_{C B}=\frac{k_{S}^{M I N} \cdot l^{3}}{192 \cdot I}, \quad \Phi(\lambda)=\frac{4 \lambda+2}{\lambda+2} .
$$

Equation 5 uses: a correction factor $\Phi(\lambda)$; a Young's modulus $E_{C B}$ in the case of a clamped beam, conditional for the nanobridge; a minimum stiffness $k_{S}^{M I N}=F_{S P} / D_{C}^{M A X}$ at the point of maximum deflection $D_{C}^{M A X}$; a span length $l$, selected earlier from the $l_{T}$ and $l_{S}$; a moment of inertia $I=\pi d^{4} / 64$ for a model shape of the nanobridge, a cylindrical beam with a section diameter $d$.

\section{Results and discussion}

\subsection{In-situ TEM indentation}

Figure 1 shows the load-unload indentation curves for two test cycles of a $60 \mathrm{~nm}$ diameter nanoscroll with TEM visualization of the process. In the first cycle, the indentation force is close to $10 \mu \mathrm{N}$ at the maximum deformation, about $20 \mathrm{~nm}$ (about a third of the diameter). In the second cycle, the force increases to $40 \mu \mathrm{N}$, while the deformation remains at about the same level. The apparent strengthening of the nanoscroll by almost four times in the second cycle seems to refer to an increasing stiffness of the contact of the nanoscroll with the non-smooth substrate, see Fig. 1(a) and inset, in other words, to the formation of a closer contact between them. In addition, the observable particles can be associated with phyllosilicate foreign granulas in the path of the electron beam. They can be located both in the plane in front of or behind the analyzed nanoscroll, as well as directly below it. In the last case, the measured stiffness will be significantly decreased.

Since the radii of the studied nanoscrolls are much smaller than their length, the load-unload curves can be processed using Eq. (1), see also Appendix. Accounting for data noisiness and the comparability of the nanoscroll

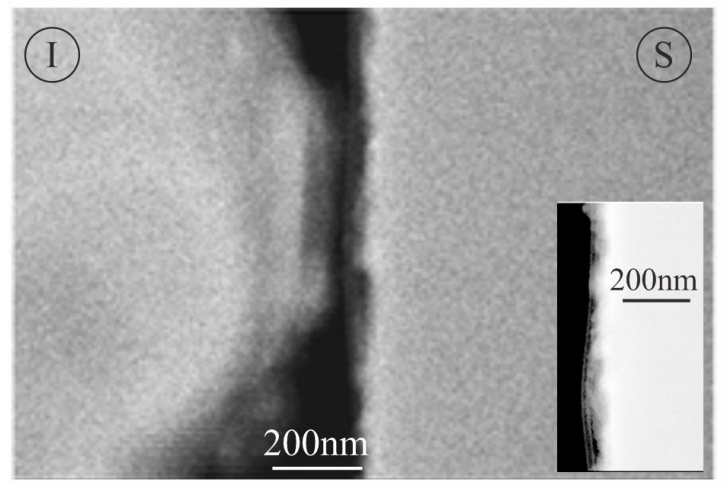

(a)

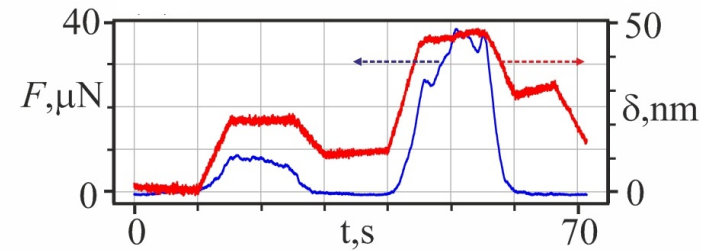

(b)

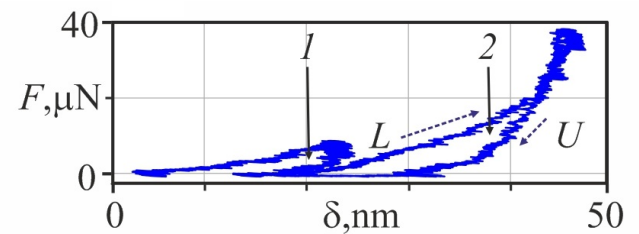

(c)

FIG. 1. (a) A STEM image of a phyllosilicate nanoscroll between the picoindenter (I) and the substrate (S) (experiment No. 7, Table 1). Inset: the STEM image of the nanoscroll-substrate interface (experiment No. 5, Table 1). (b) Measured dependences of the indentation force $F(t)$ and of the indenter displacement $\delta(t)$ on time in the experiment; (c) load (L) and unload (U) dependences $F(\delta)$. The steepest section without hysteresis (cycle 2 marked by a corresponding number and an arrow) apparently reveals the picoindenter-substrate contact 
TABLE 1. Young's moduli of the nanoscrolls, $E$, results of the in-situ TEM indentation

\begin{tabular}{|c|c|c|c|}
\hline No & $d, \mathrm{~nm}$ & $E$, according to load dependence, GPa & $E$, according to unload dependence, GPa \\
\hline 1 & 60 & Cycle 1:15; Cycle 2:50 & Cycle 1:60 \\
\hline 2 & 60 & Cycle 1:2; Cycle 2:20 & Cycle 1:10; Cycle 2:40 \\
\hline 3 & 50 & 40 & - \\
\hline 4 & 65 & 50 & 163 \\
\hline 5 & 25 & Cycle 1:12; Cycle 2:35; Cycle 3:45 & Cycle 1:55; Cycle 2:42; Cycle 3:50 \\
\hline 6 & 60 & 15 & - \\
\hline 7 & 60 & Cycle 1:10; Cycle 2:20 & Cycle 1:20; Cycle 2:35 \\
\hline
\end{tabular}

deformation with its radius, such processing gives an estimated result. Because the indentation experiment can be accompanied by inelastic processes of formation, compaction and/or elastic processes of deformation in the nanoscrollsubstrate contact, this is an underestimation.

The data of seven indentation experiments are summarized in Table 1. If each experiment is averaged over all cycles, without distinguishing between the cases of loading and unloading, and then the results of all experiments are averaged, then the final value of Young's modulus will be $40 \pm 30 \mathrm{GPa}$. Distinguishing these two cases, we get: $30 \pm 10 \mathrm{GPa}$ (load) and $60 \pm 50 \mathrm{GPa}$ (unload). A systematic increase in the value of Young's modulus calculated from the unloading section of the $F(\delta)$ dependence can also indicate in favor of creating a tighter nanoscroll-substrate contact.

\subsection{Results of bending-based tests and their comparison with in-situ TEM indentation data}

AFM data of bending-based tests of $\mathrm{Ni}_{3} \mathrm{Si}_{2} \mathrm{O}_{5}(\mathrm{OH})_{4}$ nanoscrolls obtained in PeakForce QNM mode are shown in Fig. 2. The nanoscroll formed a bridge across the grating groove, as seen in the image in Fig. 2(a). On the simultaneously measured and then corrected image of the deformation signal, Fig. 2(b), the signal is maximal in the nanobridge middle, light contrast. The large signal at the nanobridge top and bottom edges is apparent and has an instrumental origin associated with the AFM probe slippage [30]. The signal profile along the dashed line on the nanobridge is shown in Fig. 2(c), the right axis shows the deformation values.

According to the presented data, the nanobridge minimal stiffness is about $0.6 \mathrm{~N} / \mathrm{m}$, Table 2. In Fig. 2(c), the left axis, the normalized experimental profile is in optimal agreement with the model one, Eq. (4), at $\lambda=1.01$. Thus, the boundary conditions for the nanobridge are intermediate between the supported and clamped ends. The span length of the nanobridge is approximately $2.2 \mu \mathrm{m}$, and the nanoscroll height on the flat protrusions of the grating is $46 \mathrm{~nm}$. Accounting for the fixing (boundary) conditions, the calculated Young's modulus is $299 \mathrm{GPa}$.

Table 2 summarizes the bending-based test data for twenty nanobridges. Disregarding the fixing conditions, the Young's modulus averaged over the all experimental values are $120 \mathrm{GPa}$ and $110 \mathrm{GPa}$, excluding the minimum and maximum values; taking these conditions into account - $200 \mathrm{GPa}$ and $170 \mathrm{GPa}$, respectively.

In most cases, the AFM experiment shows a significantly higher value of Young's modulus than the TEM experiment does. In the both experiments, the nanoscrolls diameters do not differ on average $(54 \pm 14 \mathrm{~nm}$, Table 1 ; $56 \pm 14 \mathrm{~nm}$, Table 2). However, depending on the way of averaging, mean values of the Young's modulus in the AFM experiments are 3-6 times higher than those in the TEM experiments. Since the projected TEM image of the nanoscroll on the $1 \mu \mathrm{m}$-thick-substrate is analyzed, it is difficult to inspect the nanoscroll-substrate contact directly at the vicinity of the indentation region. If, for example, the nanoscroll covers a cavity on the substrate, then processing of the indentation curves will give an incorrect and underestimated value of Young's modulus. Let us estimate the cavity width corresponding to the stiffness values $\sim 1 \mathrm{kN} / \mathrm{m}$ in the TEM experiment, see Fig. 1. According to the data in Table 2, on average, a $2 \mu \mathrm{m}$-nanobridge has a bending stiffness of $1 \mathrm{~N} / \mathrm{m}$. The beam stiffness is inversely proportional to the cube of its length, and the nanobridge over the $200 \mathrm{~nm}$-wide-cavity will have the stiffness $\sim 1 \mathrm{kN} / \mathrm{m}$.

As shown in [7], phyllosilicate nanoscrolls with a pecoraite structure have a small positive zeta potential $<30 \mathrm{mV}$, hence, they are prone to aggregation. During the sample preparation, the substrate can become contaminated with particle aggregates, so that small particles, or even layers of such particles, which are softer than the substrate, appear under the nanoscroll. This may violate an indentation experiment condition, which requires the nanoscroll adheres 
TABLE 2. Young's modulus of the nanoscrolls, $E$, according to the AFM data of the bending-based tests processed by the algorithm of [29]

\begin{tabular}{|c|c|c|c|c|c|c|c|c|c|c|c|}
\hline No & $\begin{array}{l}k_{S}^{M I N} \\
\mathrm{~N} / \mathrm{m}\end{array}$ & $\begin{array}{c}d \\
\mathrm{~nm}\end{array}$ & $\begin{array}{c}l_{T} \\
\mathrm{~nm}\end{array}$ & $\begin{array}{l}l_{S} \\
\mathrm{~nm}\end{array}$ & $R_{T}$ & $R_{S}$ & $n$ & $\lambda$ & $\Phi(\lambda)$ & $\begin{array}{l}E_{C B} \\
\mathrm{GPa}\end{array}$ & $\begin{array}{c}E \\
\mathrm{GPa}\end{array}$ \\
\hline 1 & 0.63 & 52 & 1890 & 1980 & 0.005 & 0.003 & $2.7 \mathrm{~S}$ & 0.12 & 1.17 & 71 & 83 \\
\hline 2 & 1.04 & 53 & 1860 & 1820 & 0.008 & 0.006 & $2.3 \mathrm{~S}$ & 0.49 & 1.59 & 84 & 134 \\
\hline 3 & 0.53 & 35 & - & 1270 & - & 0.004 & $2.4 \mathrm{~S}$ & 0.40 & 1.50 & 76 & 115 \\
\hline 4 & 0.73 & 58 & - & 1390 & - & 0.004 & $2.9 \mathrm{~S}$ & 0.05 & 1.07 & 18 & 20 \\
\hline 5 & 0.89 & 62 & 2010 & 1830 & 0.010 & 0.005 & $2.0 \mathrm{~S}$ & 8.88 & 3.45 & 39 & 136 \\
\hline 6 & 2.67 & 44 & 1870 & 1890 & 0.030 & 0.006 & $2.7 \mathrm{~S}$ & 0.13 & 1.18 & 510 & 603 \\
\hline 7 & 0.61 & 46 & 2170 & 2060 & 0.003 & 0.004 & $2.2 \mathrm{~T}$ & 1.01 & 2.01 & 149 & 299 \\
\hline 8 & 0.63 & 81 & 1970 & 2370 & 0.067 & 0.004 & $2.5 \mathrm{~S}$ & 0.24 & 1.32 & 21 & 27 \\
\hline 9 & 1.05 & 37 & 1870 & 2010 & 0.013 & 0.008 & $2.2 \mathrm{~S}$ & 0.69 & 1.77 & 480 & 849 \\
\hline 10 & 0.84 & 53 & 2130 & 2030 & 0.004 & 0.005 & $2.1 \mathrm{~T}$ & 1.06 & 2.04 & 109 & 222 \\
\hline 11 & 0.54 & 52 & - & 2280 & - & 0.002 & $2.1 \mathrm{~S}$ & 1.19 & 2.12 & 93 & 196 \\
\hline 12 & 0.71 & 81 & 2120 & 1970 & 0.025 & 0.003 & $2.8 \mathrm{~S}$ & 0.09 & 1.13 & 13 & 15 \\
\hline 13 & 2.68 & 51 & 2090 & 1960 & 0.023 & 0.009 & $2.5 \mathrm{~S}$ & 0.26 & 1.35 & 318 & 427 \\
\hline 14 & 0.33 & 68 & 1940 & 1810 & 0.023 & 0.004 & $2.7 \mathrm{~S}$ & 0.14 & 1.20 & 10 & 12 \\
\hline 15 & 0.50 & 50 & 1970 & 1870 & 0.003 & 0.003 & $2.0 \mathrm{~S}$ & 2.65 & 2.71 & 56 & 151 \\
\hline 16 & 0.88 & 50 & 1910 & 1740 & 0.005 & 0.010 & $2.6 \mathrm{~T}$ & 0.17 & 1.24 & 104 & 128 \\
\hline 17 & 0.33 & 44 & 1900 & 1830 & 0.013 & 0.004 & $2.6 \mathrm{~S}$ & 0.17 & 1.24 & 57 & 70 \\
\hline 18 & 0.96 & 54 & 2060 & 1790 & 0.005 & 0.007 & $2.0 \mathrm{~T}$ & 4.02 & 3.00 & 105 & 314 \\
\hline 19 & 0.48 & 81 & 1900 & 2000 & 0.024 & 0.008 & $2.4 \mathrm{~S}$ & 0.36 & 1.46 & 9 & 14 \\
\hline 20 & 1.55 & 64 & 1920 & 2210 & 0.024 & 0.008 & $2.4 \mathrm{~S}$ & 0.30 & 1.39 & 106 & 148 \\
\hline \multicolumn{10}{|c|}{ Average Young's modulus: } & $120 \pm 150$ & $200 \pm 220$ \\
\hline \multicolumn{10}{|c|}{$\begin{array}{l}\text { Average Young's modulus across the entire sample without } \\
\text { two extreme values: }\end{array}$} & $110 \pm 120$ & $170 \pm 160$ \\
\hline
\end{tabular}

Designations used: minimum stiffness $k_{S}^{M I N}$ and diameter $d$ of the nanobridge; residual, $R_{T}$ or $R_{S}$, of the model and experimental profile and the span length, $l_{T}$ or $l_{S}$, according to the stiffness, $S$, topography, $T$; fitting parameters $n$ and $\lambda$ of model dependences (3) and (4); correction factor $\Phi(\lambda)$ for $E_{C B}$ conditional case of clamped beam, Eq.(5). 
(a)
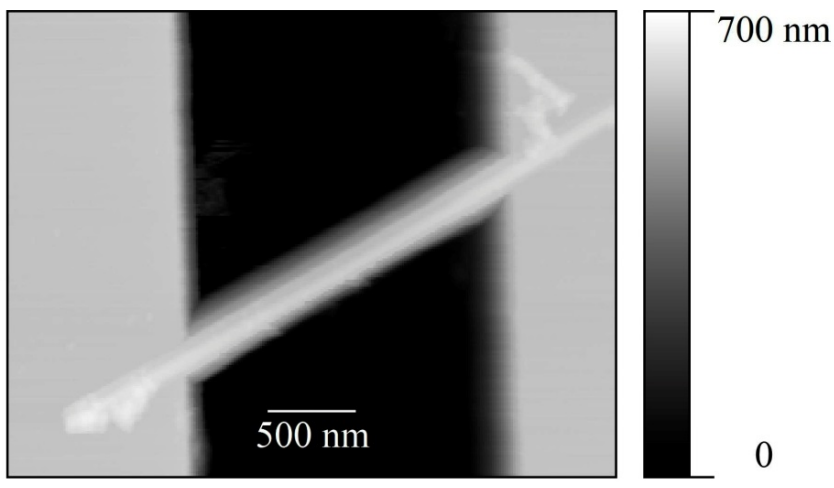

(b)

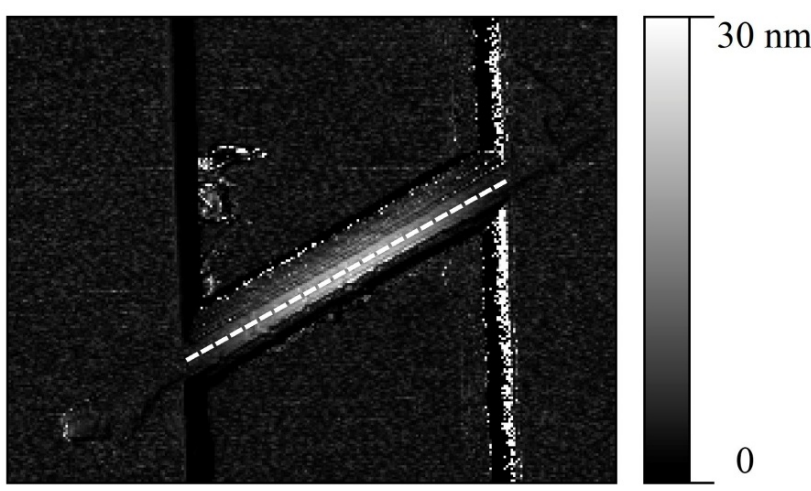

(c)

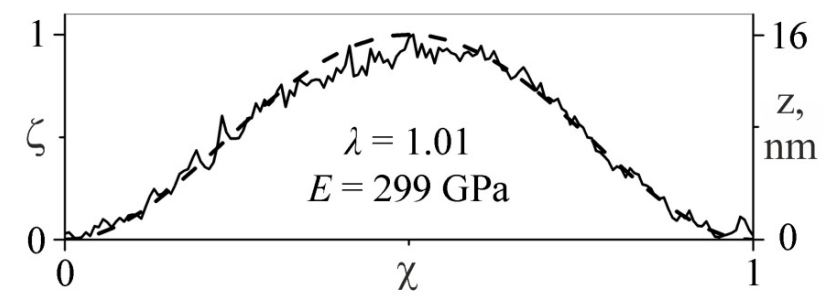

FIG. 2. AFM experiment No. 7. (a) The AFM topography image of a region of the TGZ3 grating with the $\mathrm{Ni}_{3} \mathrm{Si}_{2} \mathrm{O}_{5}(\mathrm{OH})_{4}$ nanobridge. (b) The AFM image of the $D_{C}$ signal, corrected deformation. The nanobridge bending profile data were taken along the dashed line (c). The normalized nanobridge bending profile $D_{C} / D_{C}^{M A X}$ and the model profile (dashed line), Eq. (4) with a fitting parameter $\lambda=1.01$. PeakForce QNM parameters: $F_{S P}=10 \mathrm{nN}$, Peak Force Amplitude $-150 \mathrm{~nm}$, Peak Force Frequency -1 kHz, Scan Rate -0.3 Hz, Frame Size $-256 \times 196$, cantilever FMG01 (NT-MDT SI)

tightly to the substrate. It is difficult to identify inappropriate nanoscrolls in TEM, as noted above. Such an opportunity is provided by combined SEM and AFM studies.

Two samples prepared for TEM experiments were investigated by SEM and AFM, see Fig. 3. To test the suitability of nanoscrolls for the TEM experiments, the AFM stiffness signal was analyzed. This signal measured in HybriD Mode (NT-MDT SI) corresponds to the force curve slope. On a solid and flat sample, it is maximum and is considered equal to the cantilever stiffness. The amount of the signal reduction determines the stiffness of the probe-sample contact; see more details in [30]. In Fig. 3(b), the AFM signal is maximal on the upper portion of the substrate and on a nanoscroll. Since this nanoscroll is indistinguishable from a solid substrate in terms of stiffness, it can be assumed that it adheres tightly to it. On another nanoscroll in Fig. 3(d), the signal differs (darker contrast) from the maximum by about $10 \%$. Neglecting the probe-nanoscroll contact contribution, one can estimate the nanoscroll-substrate contact stiffness as $500 \mathrm{~N} / \mathrm{m}(\cong 54 \cdot 0.9 / 0.1)$. That is close to characteristic stiffness values $\sim 1 \mathrm{kN} / \mathrm{m}$ in the in-situ TEM indentation experiments, Fig. 1.

The AFM data in Fig. 3 reproduced well in repeated scans. Consequently, they reflect elastic responses and, in particular, the elastic behavior of the probe-nanoscroll-substrate contacts. The presented results reveal very likely the elastic deformation of the nanocroll-substrate contact that might lead to underestimated values of Young's modulus 
(a)

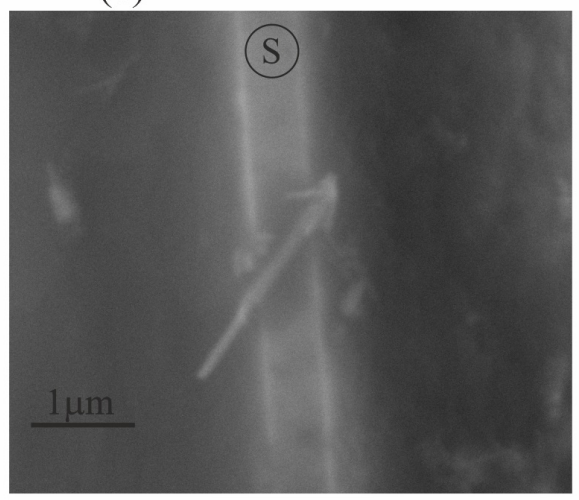

(b)

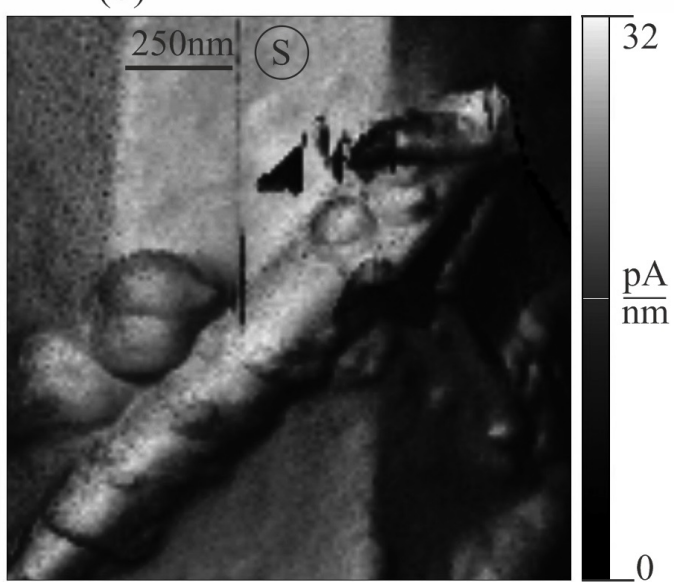

(c)

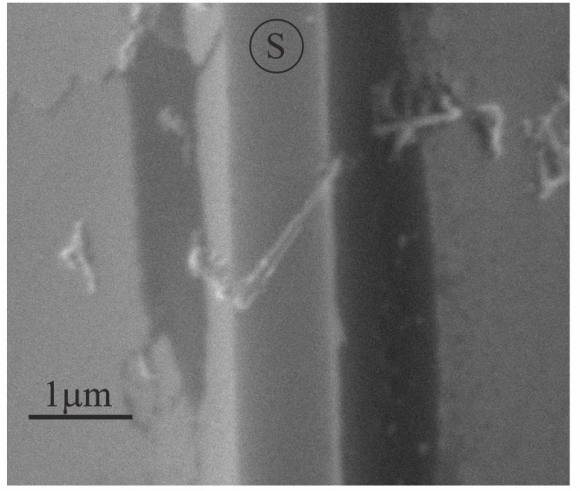

(d)

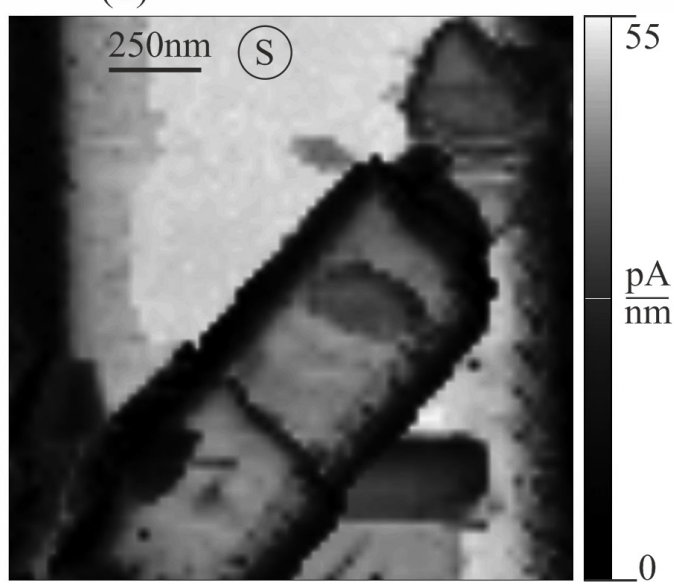

FIG. 3. (a), (c) SEM images of $\mathrm{Ni}_{3} \mathrm{Si}_{2} \mathrm{O}_{5}(\mathrm{OH})_{4}$ nanoscrolls on the substrates, $\mathrm{S}$; these substrates are also used in the TEM experiments. (b), (d) The AFM stiffness signal images of the same nanoscrolls as in (a) and (c), correspondingly. HybriD Mode parameters: (a) $F_{S P}=300 \mathrm{nN}$, cantilever stiffness calibrated by the Sader method [32] 56 N/m, Amplitude - $50 \mathrm{~nm}$, Frequency - $1 \mathrm{kHz}$, Scan Rate $0.25 \mathrm{~Hz}$, Frame Size - $128 \times 128$; (b) 150 nN, 54 N/m, 50 nm, 1 kHz, $0.2 \mathrm{~Hz}, 64 \times 64$; cantilevers RTESPA-300 (Bruker)

in the TEM experiments, Fig. 1 and Table 1. The AFM study of inelastic deformations in the nanoscroll-substrate contact may be difficult to implement, since it will require two orders of magnitude larger indentation forces, as in the TEM experiments. In conclusion it should be emphasized that the presented SEM and AFM results show the way to select the nanoscrolls for further in-situ TEM indentation measurements.

\section{Conclusions}

In this research, the Young's modulus of synthetic phyllosilicate $\mathrm{Ni}_{3} \mathrm{Si}_{2} \mathrm{O}_{5}(\mathrm{OH})_{4}$ nanoscrolls with a pecoraite structure was measured by AFM and in-situ TEM indentation techniques. For the first time, the quantitative results obtained by both the techniques were cross-compared.

In AFM experiments, advanced bending-based tests with an algorithm that identifies and takes into account the boundary conditions of nanobridges formed by the nanoscrolls were applied to measure their Young's moduli. The average Young's modulus was $200 \mathrm{GPa}$.

Additionally, the nanoscrolls were tested by modern in-situ TEM indentation technique using the Hysitron PI-95 setup. The mean measured Young's modulus value obtained was $40 \mathrm{GPa}$.

Also to analyze the indentation experiments, a convenient form of Hertz theory dependence for the contact of an elliptic paraboloid with a cylinder was proposed and used.

The nanoscrolls prepared for TEM measurements were also studied by SEM and AFM methods. Evidences of deformation of the nanoscroll-substrate contact were found. For the nanoscroll indentation experiments, this unaccounted deformation could be the main reason for the obtained low values of Young's modulus. This result seems to 
be very useful for systematizing in-situ TEM indentation measurements, as it shows that preliminary combined SEM and AFM studies allow selection of the nanoscrolls presumably weakly or strongly attached to the substrate.

\section{Acknowledgements}

This work was supported by the Russian Science Foundation grant 19-13-00151. An access to the Libra 200 TEM and Hysitron PI-95 in-situ picoindenter was provided by the M. V. Lomonosov Moscow State University Program of Development.

\section{Appendix} function

A probe (an indenter) with an elliptic paraboloid surface is described in the $X Y Z$ coordinate system by the

$$
z_{P}=\frac{x^{2}}{2 r_{x}}+\frac{y^{2}}{2 r_{y}} .
$$

The surface of the cylinder corresponding to a sample (a nanoscroll) is specified in the $X_{S} Y_{S} Z$ coordinate system by the function

$$
z_{S}^{*} \cong-\frac{x_{S}^{2}}{2 R}, \quad\left|x_{S}\right| \ll R,
$$

see Fig. 4. The principal radii of curvature of the elliptic paraboloid, $r_{x}$ and $r_{y}$, and the radius of the cylinder, $R$, are introduced. If the angle $\widehat{X, X_{S}}=\alpha$, then $x_{S}=x \cos \alpha+y \sin \alpha$, and in the $X Y Z$ coordinate system the cylinder surface is described by the function

$$
z_{S}=-\frac{\cos ^{2} \alpha x^{2}+\sin ^{2} \alpha y^{2}}{2 R} .
$$

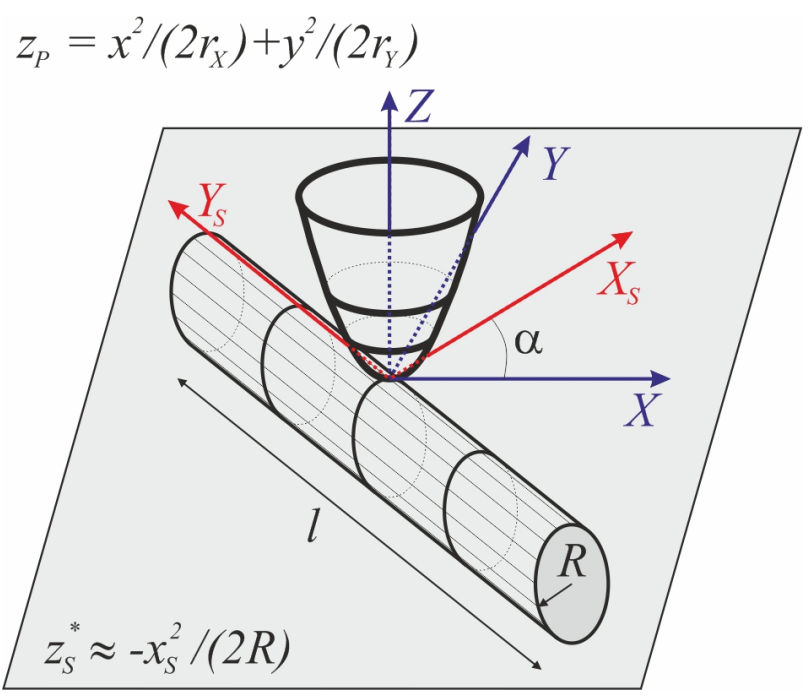

FIG. 4. The model indenter with the elliptic paraboloid surface, $z_{P}$ dependence, and the nanoscroll sample with the cylinder surface, $z_{S}^{*}$ dependence. The origin of the coordinates of both systems is at the point of contact

In the $X Y Z$ coordinate system, the distance between some point on the cylinder (sample surface of the nanoscroll, nanotube) and the point located vertically above it on the elliptic paraboloid (the indenter surface) is determined by the function $z$ (also the elliptic paraboloid):

$$
z=z_{P}-z_{S}=\frac{x^{2}}{2 R_{x}}+\frac{y^{2}}{2 R_{y}}
$$

with curvature radii at the point $x, y, z=0$ :

$$
R_{x}=\frac{R r_{x}}{R+r_{x} \cos ^{2} \alpha}, \quad R_{y}=\frac{R r_{y}}{R+r_{y} \sin ^{2} \alpha} .
$$


We also introduce the reduced radius $R^{*}$ at this point and the ellipticity parameter $\epsilon$ of the horizontal section of the surface (9) by any plane $z=$ const $>0$ :

$$
R^{*}=\frac{R_{x} R_{y}}{R_{x}+R_{y}}=\frac{R r_{x} r_{y}}{R\left(r_{x}+r_{y}\right)+r_{x} r_{y}}, \quad \epsilon=\frac{R_{y}}{R_{x}}=\frac{r_{y}\left(R+r_{x} \cos ^{2} \alpha\right.}{r_{x}\left(R+r_{y} \sin ^{2} \alpha\right)} .
$$

If the distance between the points located on the same vertical on the probe and on the sample is described by the elliptic paraboloid, then there is the following solution to the Hertz problem, see Eqs. (9) and (10) in [33]:

$$
\delta_{1}=\left[\gamma \frac{9 F^{2}}{16 E^{* 2} R^{*}}\right]^{1 / 3}, \quad \gamma=\frac{2 \mathcal{F}^{3}}{\pi^{2} k^{2} \mathcal{E}} .
$$

The exact solution of the Hertz problem for a spherical indenter probe of radius $r$ on a flat sample is obtained from (12) by substituting $\gamma=0.5$ and $R^{*}=r / 2$. The reduced Young's modulus $E^{*}$ is expressed in terms of Young's moduli $E_{P}, E_{S}$ and Poisson's ratios $\nu_{P}, \nu_{S}$ of the probe, $P$, and of the sample, $S: E^{*}=\left(\left(1-\nu_{P}^{2}\right) / E_{P}+\left(1-\nu_{S}^{2}\right) / E_{S}\right)^{-1}$; $\delta_{1}$ deformation of the contact area, $F$ force of indentation; $\mathcal{E}$ and $\mathcal{F}$ values of complete elliptic integrals of the first and second kind, $k$ ellipticity of the contact area (approximately equal to $\epsilon^{2 / 3}$ ), [33]. In [33], see equations (6)-(8), analytical dependences were obtained that approximately relate $k, \mathcal{E}$ and $\mathcal{F}$ with the ellipticity parameter $\epsilon$ :

$$
\begin{gathered}
k \cong 1.0339 \epsilon^{0.636}, \\
\mathcal{E} \cong 1.0003+0.5968 \epsilon^{-1}, \\
\mathcal{F} \cong 1.5277+0.6023 \ln \epsilon, \\
k^{2} \mathcal{E} \cong 1.0693 \epsilon^{1.272}+0.6379 \epsilon^{0.272} .
\end{gathered}
$$

Using (15) and (16), we obtain an approximate dependence for $\gamma$ from (12):

$$
\gamma=\frac{2(1.5277+0.6023 \ln \epsilon)^{3}}{\pi^{2}\left(1.0693 \epsilon^{1.272}+0.6379 \epsilon^{0.272}\right)} .
$$

For a spherical indenter on a flat sample $\epsilon=1$, and from (13-15) and (17) we obtain: $\mathcal{E}(1) \cong 1.5971, \mathcal{F}(1) \cong$ $1.5277, k(1) \cong 1.0339$, and $\gamma=2 \times 1.5277^{3} /\left(\pi^{2} 1.0339^{2} 1.5971\right) \cong 0.4232<0.5$. Substitution of (15) in (12) in the case $\varepsilon=1$ underestimates the indentation value $\left(\delta_{1} / \delta_{H}=\sqrt[3]{0.4232 / 0.5} \cong 0.95\right)$ in comparison with the exact Hertz solution by $5 \%$. As shown in [33], in the other cases the error is smaller.

To calculate the values of $E^{*}$ in the in-situ TEM indentation experiment, the indentation curves can be analyzed using the simplified expression (17) for $\gamma$ in (12):

$$
\gamma \approx \frac{2(1+0.4 \ln \epsilon)^{3}}{\epsilon^{0.27}(3 \epsilon+1.8)}
$$

Considering the picoindenter (probe tip) as a paraboloid of revolution with a radius of curvature $r$, and a nanoscroll as a cylinder with a radius of curvature $R$, we have:

$$
R^{*}=\frac{R r}{2 R+r} \quad \text { and } \quad \epsilon=\frac{R+r}{R} .
$$

$R^{*}$ differs from the reduced contact radius of two balls with radii $r$ and $R(=R /(R+r))$.

The experimental force curves can be characterized, for example, by the stiffness of the picoindenter-nanoscrollsubstrate composite system. Eq. (12), taking into account (18) and (11), makes it possible to determine the stiffness of the picoindenter-nanoscroll contact $k_{1}$ :

$$
k_{1}=\frac{\partial F}{\partial \delta_{1}}=2 E^{*} \sqrt{R^{*} \delta_{1} / \gamma}
$$

In the TEM experiment, the total deformation is measured: $\delta=\delta_{1}+\delta_{2}$. Where $\delta_{1}$ and $\delta_{2}$ are the deformations of the picoindenter-nanoscroll contact and of the nanoscroll-substrate contact, see also Fig. 4. It is possible to determine $k_{2}$ as the stiffness of a cylinder of length $l$ with a substrate with Young's modulus $E_{S b}$ and Poisson's ratio $\nu_{S b}$. It does not depend on the cylinder radius $R,[24,34]$ :

$$
F=\frac{\pi}{4} E^{* *} l \delta_{2}, \quad E^{* *}=\left(\frac{1-\nu_{S}^{2}}{E_{S}}+\frac{1-\nu_{S b}^{2}}{E_{S b}}\right)^{-1}, \quad k_{2}=\frac{\partial F}{\partial \delta_{2}}=\frac{\pi}{4} E^{* *} l .
$$

Assuming $E^{*} \sim E^{* *}$, the deformation $\delta_{2}$ can be ignored in the experiment when $\pi l \gg 8 \sqrt{R^{*} \delta_{1} / \gamma}$. Since $\delta_{1} \leq \delta \leq R$, and $\gamma \sim 1$, the condition is simplified to $l \gg R^{*}$. 


\section{References}

[1] Krasilin A.A., Khrapova E.K., Maslennikova T.P. Cation Doping Approach for Nanotubular Hydrosilicates Curvature Control and Related Applications. Crystals, 2020, 10(8), P. 654(1-41).

[2] Krasilin A.A. Energy modeling of competition between tubular and platy morphologies of chrysotile and halloysite layers. Clays Clay Miner., 2020, 68, P. 436-445.

[3] Prinz V.Ya., Seleznev V.A., Gutakovsky A.K., Chehovskiy A.V., Preobrazhenskii V.V., Putyato M.A., Gavrilova T.A. Free-standing and overgrown InGaAs/GaAs nanotubes, nanohelices and their arrays. Physica E: Low-dimensional Systems and Nanostructures, 2000, 6, P. 828831.

[4] Gulina L.B., Tolstoy V.B., Lobinsky A.A., Petrov Yu.V. Formation of Fe and $\mathrm{Fe}_{2} \mathrm{O}$ Microspirals via Interfacial Synthesis. Part. Part. Syst. Charact., 2018, 35, P. 1800186

[5] Scarfato P., Avallone E., Incarnato L., Di Maio L. Development and evaluation of halloysite nanotube-based carrier for biocide activity in construction materials protection. Appl. Clay Sci., 2016, 132, P. 336-342.

[6] Cavallaro G., Danilushkina A., Evtugyn V., Lazzara G., Milioto S., Parisi F., Rozhina E., Fakhrullin R. Halloysite Nanotubes: Controlled Access and Release by Smart Gates. Nanomaterials, 2017, 7(8), 199, P. 1-12.

[7] Krasilin A.A., Danilovich D.P., Yudina E.B., Bruyere S., Ghanbaja J., Ivanov V.K. Crystal violet adsorption by oppositely twisted heat-treated halloysite and pecoraite nanoscrolls. Appl. Clay Sci., 2019, 173, P. 1-11.

[8] Zhang C., Zhu W., Li S., Wu G., Ma X., Wang X., Gong J. Sintering-resistant Ni-based reforming catalysts obtained via the nanoconfinement effect. Chem. Commun., 2013, 49, P. 9383(1-3).

[9] Ashok J., Bian Z., Wang Z., Kawi S. Ni-phyllosilicate structure derived Ni-SiO $2-\mathrm{MgO}$ catalysts for bi-reforming applications: acidity, basicity and thermal stability. Catal. Sci. Technol., 2018, 8(6), P. 1730-1742.

[10] Bian Z., Kawi S. Highly carbon-resistant $\mathrm{Ni}-\mathrm{Co} / \mathrm{SiO}_{2}$ catalysts derived from phyllosilicates for dry reforming of methane. J. CO 2 Util., 2017, 18, P. 345-352.

[11] Krasilin A.A., Straumal E.A., Yurkova L.L., Khrapova E.K., Tomkovich M.V., Shunina I.G., Vasil'eva L.P., Lermontov S.A., Ivanov V.K. Sulfated Halloysite Nanoscrolls as Superacid Catalysts for Oligomerization of Hexene-1. Russ. J. Appl. Chem., 2019, 92(9), P. $1251-1257$.

[12] Park J.C., Kang S.W., Kim J.-C., Kwon J.I., Jang S., Rhim G.B., Kim M., Chun D.H., Lee H.-T., Jung H., et al. Synthesis of Co/SiO 2 hybrid nanocatalyst via twisted $\mathrm{Co}_{3} \mathrm{Si}_{2} \mathrm{O}_{5}(\mathrm{OH})_{4}$ nanosheets for high-temperature Fischer-Tropsch reaction. Nano Res., $2017, \mathbf{1 0}(3)$, P. 1044-1055.

[13] Liu Y., Liu M. Conductive carboxylated styrene butadiene rubber composites by incorporation of polypyrrole-wrapped halloysite nanotubes. Compos. Sci. Technol., 2017, 143, P. 56-66.

[14] Afanas'eva N.V., Gubanova G.N., Romashkova K.A., Sapegin D.A., Kononova S.V. Relaxation processes in an aromatic polyamide-imide and composites on its basis with hydrosilicate nanoparticles. Polym. Sci. Ser. A, 2016, 58(6), P. 956-967.

[15] Mo H., Yang K., Li S., Jiang P. High thermal conductivity and high impact strength of epoxy nanodielectrics with functionalized halloysite nanotubes. RSC Adv., 2016, 6(73), P. 69569-69579.

[16] Roy K., Debnath S.C., Pongwisuthiruchte A., Potiyaraj P. Up-to-date review on the development of high performance rubber composites based on halloysite nanotube. Appl. Clay Sci., 2019, 183, P. 105300(1-15).

[17] Binnig G., Quate C.F., Gerber Ch. Atomic Force Microscope. Phys. Rev. Lett., 1986, 56(9), P. 930-933.

[18] Wong E.W., Sheehan P.E., Lieber C.M. Nanobeam Mechanics: Elasticity, Strength, and Toughness of Nanorods and Nanotubes. Science, 1997, 277(5334), P. 1971-1975.

[19] Bhushan B. edd. Nanotribology and Nanomechanics - An Introduction. Springer-Verlag Berlin Heidelberg, 2005,1148 p.

[20] Salvetat J.-P., Kulik A.J., Bonard J.-M., Briggs G.A.D., Stockli T., Méténier K., Bonnamy S., Béguin F., Burnham N.A., Forró L. Elastic Modulus of Ordered and Disordered Multiwalled Carbon Nanotubes. Adv. Mater., 1999, 11(2), P. 161-165.

[21] Salvetat J.-P., Bonard J.-M., Thomson N.H., Kulik A.J., Forró L., Benoit W., Zuppiroli L. Mechanical properties of carbon nanotubes. Appl. Phys. A, 1999, 69(3), P. 255-260.

[22] Zheng X.-P., Cao Y.-P., Li B., Feng X.-Q., Wang G.-F. Surface effects in various bending-based test methods for measuring the elastic property of nanowires. Nanotechnology, 2010, 21(20), P. 205702(1-6).

[23] Timoshenko S., Goodier J.N. Theory of elasticity. McGraw-Hill, New York, 1970, 567 p.

[24] Landau L.D., Lifshitz E.M. Theory of Elasticity. Pergamon Press Ltd., Oxford, 1970, 165 p.

[25] Cuenot S., Demoustuer-Champagne S., Nysten B. Elastic modulus of polypyrrole nanotubes. Phys. Rev. Lett., 2000, 85(8), P. 1690-1693.

[26] Kis S. Mechanical properties of mesoscopic objects. Thesis. Ecole Polytechnique Federale de Lausanne, 2003,166 p.

[27] Bruker Hysitron nanomechanical test instruments. URL: https:/www.bruker.com/ products/surface-and-dimensionalanalysis/nanomechanical-test-instruments.html

[28] Khrapova E.K., Ugolkov V.L., et al. Thermal behavior of Mg-Ni-phyllosilicate nanoscrolls and performance of the resulting composites in hexane-1 and acetone hydrogenation. Chem. Nano. Mat., 2020, P. 1-14.

[29] Ankudinov A.V. A New Algorithm for Measuring the Young's Modulus of Suspended Nanoobjects by the Bending-Based Test Method of Atomic Force Microscopy. Semiconductors, 2019, 53(14), P. 1891-1899.

[30] Ankudinov A.V., Khalisov M.M. Contact Stiffness Measurements with an Atomic Force Microscope. Technical Physics, 2020, 65(11), P. $1866-1872$

[31] Nečas D., Klapetek P. Gwyddion: an open-source software for SPM data analysis. Cent. Eur. J. Phys., 2012, 10(1), P. 181-188.

[32] Sader J.E., Chon J.W.M., Mulvaney P. Calibration of rectangular atomic force microscope cantilever. Rev. Sci. Instrum., 1999, 70, P. 39673969

[33] Brewe D.E., Hamrock B.J. Simplified solution for elliptical contact deformation between two elastic solids. J. Lubrication Technology, 1977, 99 (4), P. 485-487.

[34] Popov V.L., Heß M., Willert E. Handbook of Contact Mechanics. Exact Solutions of Axisymmetric Contact Problems. Translation from the German Language edition: Popov et al: Handbuch der Kontaktmechanik. Springer-Verlag GmbH Deutschland, 2018,347 p. 